\author{
А.Б. БАНОВАЦ
}

\title{
Топологическое представление материальной импликации и правила вывода modus ponens ${ }^{1}$
}

\author{
Бановац Андрей Борисович \\ Институт философии РАН. \\ 119991, Российская Федерация, Москва, ул. Волхонка, д. 14, стр. 5. \\ E-mail: pochtamoya@bk.ru
}

В настоящей статье вводятся и обосновываются некоторые основные элементы методологического подхода, характеризуемого применением топологических средств анализа и представления сущностей посредством актуализации произвольной явной схемы дифференциации (схемы различения). Последняя понимается в качестве множества правил, именуемых критериями дифференциации, выделяющих специфические инварианты (симметрии) исследуемой сущности. Вводится понятие инвариантов дифференциации как символических представителей последних и показывается, что во всех случаях проведения дифференциации на множестве упомянутых инвариантов может быть индуцирована топологическая структура. Отправляясь от полученных результатов, в дальнейшем даем теоретические рамки представления и обращения с объектами логических теорий и систем, а также представления их свойств и взаимоотношений с использованием формальных средств топологии, с целью их описания в терминах топологических свойств. Изложение предложенного метода дано через его применение, в качестве результата которого получается топологическое представление материальной импликации и правила вывода modus ponens.

Ключевые слова: дифференциация, инварианты дифференциации, дельта-функция Кронекера, топологическое пространство, топологическое представление, топологические инварианты, материальная импликация, modus ponens

\section{1. Введение}

В цикле своих работ по анализу пространственности и времени применительно к теории и философии искусства П.А. Флоренский пишет: «... Проблема пространства залегает в средоточии миропонимания во всех возникавших системах мысли и предопределяет сложение всей системы. С известными ограничениями и разъяснениями можно было бы даже признать пространство за собственный и первичный предмет философии, в отношении к которому все прочие философские темы приходится оценивать как производные. И чем плотнее сработана та или

\footnotetext{
${ }^{1}$ Работа выполнена при поддержке РГНФ, проект № 13-03-00384.
} 
другая система мысли, тем определеннее ставится в качестве ее ядра своеобразное истолкование пространства. Повторяем: миропонимание - пространствопонимание» [4, с. 272]. И далее: «Вся культура может быть истолкована, как деятельность организации пространства» [3, c. 112].

Развивая эти тезисы применительно к онтологии, гносеологии, культурологическим исследованиям и теории искусства, П.А. Флоренский - кроме прочего - связывает смысловую составляющую понятия и действия с их предполагаемыми пространственными особенностями как ключевыми. Анализ последних, в его трактовке, осуществляется применением математического формализма, включающего элементы математического анализа и дифференциальной геометрии. Один из моментов, наглядно представляющий общие черты его методологического приема, находим на первых страницах [3] в проведенном при использовании упомянутых формальных элементов рассмотрении гносеологического значения и ценности перехода из пространства, характеризуемого классической геометрией в пространство, геометрические свойства которого определены конечным, положительным значением кривизны [3, c. 95-104].

Далее, отправляясь именно от концепции пространственности о. Павла, изложенной выше в ее общих чертах, мы изложим некоторые принципы, с помощью которых можно строить топологические представления логических объектов.

Свойства полученных представлений, на наш взгляд, открывают новые возможности для обращения с последними, включая моделирование взаимосвязей между ними, между их семантическим и синтаксическим аспектами, осуществление формальных переходов между логическими системами вообще, а также новый способ их характеризации на общих топологических основаниях.

С другой стороны, по нашему мнению, в рамках упомянутого подхода возникают и новые методологические возможности для логической формализации топологически описуемых объектов, а как будет видно из дальнейшего - любая сущность, в той или иной степени, позволяет такое описание. Именно эта, вторая особенность принципов, о которых пойдет речь, позволяет строить логические формы, отношения и переходы между ними как обладающие релевантным характером, и - конструктивно. Из сказанного понятно, что мы стремимся к построению метода который, между прочим, позволяет: 
1) представить логические теории и системы в качестве топологических объектов и их отношений, т.е. позволяет перевести на топологические основания объектный уровень данного формального языка;

2) описывать логические теории и системы на метауровне и осуществлять формальные переходы из одних теорий или систем в другие с использованием топологического формализма.

Именно особенности этих переходов - отображений между предполагаемыми классами топологических объектов, по нашему мнению, предоставят возможность характеризации логических систем в терминах топологических инвариантов их представлений.

В данной статье мы не пытаемся дать сколько-нибудь законченную конструкцию предлагаемого приема, а лишь предварительно очертить его контуры и через приложения, касающиеся топологического представления некоторых логических объектов, представить эффективность его методологических принципов.

Как уже понятно, формальную основу для изложенного метода составляет топологический аппарат. Именно уровень обобщения понятия пространства в топологии, с одной стороны, и применимость этого обобщения по отношению к возможностям моделирования объектов с другой, представляется нам достаточно формально сильным и в то же время, достаточно емким моделирующим средством, позволяющим структурировать модели объектов разного уровня, не искажая формальными предпосылками своего применения те свойства моделируемого объекта, которые для нас представляются существенными.

Начнем наше рассмотрение с описания дифференциации как необходимого условия познания, принцип действия которого по отношению к объекту результирует формированием его мысленной репрезентации у субъекта. На уровне рудиментарного символического представления последнюю можно рассматривать в качестве топологического пространства.

Поскольку дифференциации подлежат и формальные объекты, в том числе и сама логическая форма, определенная своими семантическими и синтаксическими характеристиками, последняя тоже допускает топологическое описание. Этот момент нам кажется особенно важным, поскольку, по нашим соображениям, топологическое описание логических систем через дифференциацию позволяет трактовку на объектном уровне и метауровне в рамках одной формальной схемы (пункт 2). 
Именно на примере топологического описания материальной импликации, правила вывода modus ponens и комментариях к последним мы попытаемся показать как можно осуществить упомянутое. Одновременно обоснуем тезисы о возможностях применения данного приема для установления связей между различными логическими системами, использующими различные связки или различные их определения, а также различные уровни анализа логической формы объектов.

\section{2. Дифференциация как необходимое условие познания сущностей. Необходимые условия дифференциации}

Рецепция какой-либо сущности на каком-либо уровне сознания предполагает определенную возможность различения данной сущности по отношению к тому, что ею не является. Способность различения сущностей вообще будем называть дифференциацией, и в этом параграфе покажем, что именно дифференциация представляет необходимое условие познания.

Явно выраженные механизмы дифференциации среды обитания присущи всем видам живых существ, начиная с вирусов и одноклеточных организмов. Наличие этих механизмов и, следовательно, дифференциация физической среды представляет необходимое условие их существования, поскольку в отсутствие данной возможности организмы не были бы в состоянии различать пригодную для жизни среду от непригодной (по ряду существенных параметров).

Когда речь идет о более развитых организмах, механизмы дифференциации усложняются в процессе развития и дифференцирования органов чувств, а у человека эти механизмы включают и присущую ему когнитивную систему, позволяющую, между прочим, осуществлять рефлексию, т.е. дифференциацию мысли. В последнем случае существенную роль играет природный язык, чья феноменальность подразумевает дифференциацию фонетических и морфологических элементов, наделенных знаковыми функциями. В свою очередь, именно возможность (и соответственно способность) различения (дифференциации) является необходимым условием функционирования знака как в семантическом, так и в синтаксическом плане ${ }^{2}$.

Язык выступает как один из важнейших инструментов дифференциации сущностей, которая проводится посредством категориальных схем выражения, и в этом раскрывается его аналитическая и познава-

\footnotetext{
${ }^{2}$ Обоснование этого тезиса находим в работах де Соссюра, Якобсона, Трнки (см. [6, c. 340]).
} 
тельная функция. Но даже если согласимся с утверждением Витгенштейна, что «ни о какой деятельности сознания, чем-либо отличимой от употребления языка, говорить не имеет смысла» - вряд ли можно настаивать на том что язык представляет необходимое условие для осуществления дифференциации вообще. Как мы видели выше, способность различения (дифференциации) присуща и организмам, явно не обладающим языковыми компетенциями. То есть приведенные выше рассуждения представляют способность дифференциации как первичную по отношению к естественным языкам.

Неоспоримой нам кажется предпосылка, согласно которой дифференциация аспектов какой-либо сущности является необходимым условием ее восприятия на некотором уровне сознания. Дифференциация является необходимым условием для перцепции и рецепции; именно в силу ее наличия (в зависимости от ее модусов) любая подходящая сущность становится воспринимаемой в условиях чувственно-когнитивных данностей познающего субъекта. Таким образом, дифференцируемость любой сущности равносильна ее феноменальности. Отсюда следует, что дифференциация представляет необходимое условие познания.

В силу ограничений спектров чувствительности органов чувств и ограниченности когнитивного аппарата аспекты каждой сущности, представляющие область приложения дифференциации, должны обладать определенной инвариантностью по отношению к изменениям условий их наблюдения и по отношению к процессам их фиксации на определенном уровне сознания. Иными словами, необходимое условие для дифференциации феноменальных аспектов сущностей на некотором уровне сознания представляется в виде симметрий (см. [9]), т.е. инвариантностей этих аспектов по отношению к определенному типу преобразований, (непременно) происходящих в процессе их наблюдения и фиксации.

\section{3. Формальное описание дифференциации - дифференциация как пространствообразующая функция}

Введем понятие схема дифференциачии, имея пока в виду любую структуру, состоящую из неких явных критериев (проведения) дифференииации определенной сущности и отношений между ними. Различение между явными и неявными критериями условно и зависит от конкретного случая дифференциации. 
Так, например, при изучении какого-либо явления, моделируемого в рамках определенной аксиоматической научной теории, явные критерии дифференциации, в общем случае, даны основными и производными понятиями, принципами (аксиомами) данной теории и выведенными из последних утверждениями, т.е. элементами, выражающими ее аксиоматическую структуру.

$\mathrm{K}$ неявным критериям можно отнести череду факторов, в том числе и психо-физических, которые обусловливают более широкий контекст отношения субъекта к дифференцируемой сущности, т.е. таких, которые определяются уровнем восприятия, эстетическими предпочтениями, конкретными целями рассмотрения и т.д. и отражаются в структуре модели сущности, сформированной в итоге ее дифференциации.

Из сказанного следует, что определенный фрагмент реальности, взятый в качестве объекта дифференциации, может быть дифференцирован по-разному, в зависимости от того, какая схема дифференциации при этом применяется.

При наличии даже самого простого уровня механизмов дифференциации и области ее приложения в виде сущностей, обладающих сооответствующего типа феноменальными аспектами, дифференциация может быть формализована посредством д-функции Кронекера:

$$
\text { Для любых } x, y ; \partial(x, y)=\left\{\begin{array}{c}
1 ; x=y \\
0 ; x \neq y
\end{array}\right.
$$

где $x$ и $y$, в данном формальном представлении, пробегают по определенному множеству знаков, фиксирующих в терминах некоторой системы выражения те аспекты дифференцируемой сущности, которые выявляются в результате применения определенной схемы дифференциации. Данные фиксирующие элементы, поставленные в соотношение с дифференцируемыми аспектами сущности, будем называть инвариантами дифберенииации.

Поскольку данная функция удовлетворяет аксиомам метрики, то ею индуцировано метрическое пространство на множестве представлений дифференцируемых аспектов данной сущности; а поскольку любое метрическое пространство, в свою очередь, индуцирует пространство топологическое, то данное формальное представление дифференциации ставит в соответствие представлению в терминах инвариантов дифференциации каждой дифференцируемой сущности определенное топологическое пространство. 
Заметим, что данное формальное описание так же интуитивно, как и само понятие дифференциации. Далее, симметрия как необходимое условие дифференциации и ее формального представления в виде (1) отражается в топологической структуре, определяя ее структурные инварианты (элементы и их отношения). Заметим еще и то, что в данном случае, с формальной точки зрения, топологическое пространство получается конструктивно и в общем зависит, в силу применяемых критериев дифференциации, от «индивидуальной объективной реальности» субъекта [1, с. 173].

Таким образом, данное формальное описание дифференциации как фундаментальное, необходимое начало любой познавательной деятельности представляет ее как активность, образующую и организующую пространство в смысле определения топологической структуры выражения соответствующих сущностей.

Топологический характер и свойства, вытекающие из приведенной формализации дифференциации, представляют, на наш взгляд, формальное обоснование пространственной концепции П.А. Флоренского, которую О.И. Генисаретский характеризует как «Опознание пространственности в качестве первичной моделирующей системы...» ${ }^{3}$.

\section{4. Дифференциация феноменов мысли. Топологическое (мета)описание логики}

Как уже было отмечено во введении, мысленные комплексы тоже подлежат дифференциации в вышеуказанном смысле. Приведенная формализация дифференциации элементарным - формально и интуитивно ясным образом наделяет мысль как объект дифференциации пространственной структурой, позволяющей проводить дифференциацию ее аспектов в терминах топологического формализма. Тем самым мы получаем важное методологическое средство для формального метаописания функции различения, присущей языку и являющейся необходимым условием для выражения мысли во всех последующих логических системах. Заметим, что данный подход, следуя мысли о. Павла,

\footnotetext{
${ }^{3}$ Как утверждает О.И. Генисаретский, комментируя тезисы о. Флоренского: «Мы усматриваем здесь одно из важнейших созначений понимающего отношения к действительности, связывающего способность понимания с пространством как собственным и первичным предметом, условием, с одной стороны, и средством смыслодеятельности понимания, с другой... всякое понимание основано на пространствопонимании. О чем бы ни шла речь, - о мире в целом или каком-то частном предмете, о знаке или лице, образе или ценности - понять, значит понять в каком-то пространстве, в качестве пространства, посредством пространства» [2, с. 12].
} 
приведенной в самом начале, действительно позиционирует логику как «философскую тему», производную по отношению к пространству как «... собственному и первичному предмету философии» [4, с. 272].

Далее приведем пример, указывающий, как полученные результаты могут быть использованы для репрезентации и метаописания некоторых фрагментов логических систем.

\section{1. «Язык переменных» В.И. Шалака}

Формальная структура, подходящая в качестве примера для наглядного представления вышесказанного, дана «Языком переменных» В.И. Шалака [5, с. 23]. Необходимое, но недостаточное условие для данного языка, представляет дифференциация переменных, индуцирующая на синтаксическом уровне дифференциацию их символов, входящих в алфавит. Обозначим через Var множество символов переменных в данном языке. Определим на Var $\partial$-функцию Кронекера. Данная формализация структурирует множество Var как дискретное топологическое пространство (элементами которого являются символы переменных).

\section{2. Топологическая репрезентация протологического следования}

Пусть $\Sigma$ - множество выражений языка переменных [5, с. 23]. В данном «языке» приведенные В.И. Шалаком свойства протологического следования опираются ${ }^{4}$ на отношение «быть элементом множества» $\Sigma$ (свойства 1-4). Таким образом, $X$ протологически следует из $\Sigma$ если и только если расстояние между (точкой) $X$ и множеством $\Sigma$ нулевое. Отсюда понятно, что введение протологического следования, индуцирующего логическую структуру $(\operatorname{Var}, \mid=)^{5}$ на множестве $V a r$, представляет собой топологическое преобразование соответствующего дискретного топологического подпространства на $\operatorname{Var}$ в пространство с антидискретной топологией $\{\Sigma, \emptyset\}$.

В случае если $X$ не является элементом $\Sigma, X$ протологически не следует из $\Sigma$, т.е. растояние $X$ от $\Sigma$ не нулевое, топологическое пространство, индуцированное $\Sigma$, и $\{X\}$ представляет дискретное подпространство пространства $\operatorname{Var}$ с топологией $\{\Sigma \cup\{X\}, \Sigma,\{X\}, \emptyset\}$.

Иными словами, формализация дифференциации выражений языка переменных приводит к присвоению множеству переменных опреде-

\footnotetext{
${ }^{4}$ В смысле необходимости.

5 Здесь символ $\mid=$ обозначает протологическое следствие В.И. Шалака в случае «Языка переменных».
} 
ленной топологической структуры - дискретной топологии, индуцирующей на любом подмножестве выражений дискретное топологическое подпространство исходного пространства.

Наделение множества $\operatorname{Var}$ операцией протологического следования приводит к структуре $(\operatorname{Var},=)$, которую можно рассматривать в качестве предельного случая языка («Языка переменных» $[5, \text { с. 23] })^{6}$.

В связи с этим для нас является чрезвычайно важным подчеркнуть тот факт, что переход от множества $\operatorname{Var}$ к (прото)логической структуре на этом множестве может быть характеризован топологически, причем наделение множества $\operatorname{Var}$ отношением протологического следования сопровождается преобразованием топологии соответствующих его подмножеств из дискретной (в случае неструктурированного множества $V a r)$ в антидискретную (в случае $\operatorname{Var}$ как/прото/логической структуры).

Отметим еще один очевидный и интересный момент данного только что топологического (мета)описания. Поскольку в приведенном примере с протологическим следованием, определенным на некотором множестве, логические свойства могут быть однозначно характеризованы топологическими свойствами, топологические пространства или их определенные сегменты, гомеоморфные топологическому представлению языка переменных, могут быть истолкованы в соответствующих логических категориях.

\section{3. Топологическое описание материальной импликации и правила вывода modus ponens}

В дальнейшем покажем способ объединения семантических и синтаксических свойств формальных языков в рамках топологического формализма. Приведем примеры такого объединения относительно элементов логики высказываний. Основной замысел состоит в том, чтобы, рассматривая материальную импликацию как отношение между пропозициональными формулами, выявить свойства формул, образованных ею в качестве главной связки, и выразить эти свойства в терминах топологических инвариантов соответствующих топологических пространств. Вслед за этим представим топологическую характеризацию правила вывода modus ponens как гомеоморфного отображения полученных топологических пространств.

\footnotetext{
${ }^{6}$ Хотя и, как утверждает В.И. Шалак, с большой натяжкой.
} 
Tеорема $1 .{ }^{7}$ Пусть $\left(X, \mathcal{T}_{X}\right)$ - топологическое пространство и $U, V-$ собственные подмножества $X$, такие что $U=\operatorname{Int} U \neq C l U, V=$ $C l V \neq I n t V$.

Тогда существует взаимно однозначное отображсение $\psi$ множества $\mathcal{T}_{Y} \cup \mathcal{F}_{Y}$ топологически связного пространства $\left(Y, \mathcal{T}_{Y}\right), Y<X$, индуцированного множествами $U$ u $V$, на граф булевой функиии формулы логики высказываний $P \rightarrow Q(P$ и $Q$ - различные формулы), такой, что все образы открытых множеств при отображении $\psi$ соответствуют одинаковому значению булевой функиии данной формуль.

ДоКАЗАТЕЛЬСтво. Определим $Y=U \cup V$ и сформируем множество $\mathcal{T}_{Y}=\{U \cup V, U, \emptyset\}$. Очевидно $Y=X \cap Y, U \in \mathcal{T}_{X} ; U=U \cap Y$, т.е. $\left(Y, \mathcal{T}_{Y}\right)$ является топологическим подпространством $\left(X, \mathcal{T}_{X}\right)$ и $\left(Y, \mathcal{T}_{Y}\right)$ топологически связное пространство. Семейством замкнутых множеств топологического пространства $Y$ является $\mathcal{F}_{Y}=\{U \cup V, V, \emptyset\}$.

Определим отображение $\varphi: \mathcal{T}_{Y} \cup \mathcal{F}_{Y} \rightarrow \mathcal{P}(Y) \times\{0,1\}$ такое, что

$$
\varphi(A)= \begin{cases}(A, 1), & A \in \mathcal{T}_{Y} \\ (A, 0), & A \notin \mathcal{T}_{Y} .\end{cases}
$$

Множеством значений $\varphi$ является

$$
\operatorname{Im} \varphi=\{(U \cup V, 1),(U, 1),(V, 0),(\emptyset, 1)\},
$$

$\varphi$ является взаимно однозначным отображением множеств $\mathcal{T}_{Y} \cup \mathcal{F}_{Y}$ и $\operatorname{Im} \varphi \subseteq\left(\mathcal{T}_{Y} \cup \mathcal{F}_{Y}\right) \times\{0,1\}$.

Каждому элементу $R_{k} \in \operatorname{Im} \varphi ; k=\overline{1,4}$ сопоставляем отображение $\chi_{k}:\left(\mathcal{T}_{Y} \cup \mathcal{F}_{Y}\right) \backslash\{Y, \emptyset\} \rightarrow\{0,1\}$, определенное следующим образом:

$\chi_{k}(B)=1$, в случае если существует $D \in R_{k}$ такое, что $B \subseteq D$, и $\chi_{k}(B)=0$ в противном случае.

Образы соответствующих элементов множества $\left(\mathcal{T}_{Y} \cup \mathcal{F}_{Y}\right) \backslash\{Y, \emptyset\}$, сопоставленные элементам $\operatorname{Im} \varphi$ (обозначенным в порядке, который имеется в (2), через $\left.R_{1}, R_{2}, R_{3}, R_{4}\right)$, суть:

$$
\chi_{1}(U)=1 ; \quad \chi_{1}(V)=1 ;
$$

\footnotetext{
${ }^{7}$ Тот факт, что некоторое множество $M$ является топологическим пространством будем записывать, как принято, $\left(M, \mathcal{T}_{M}\right)$, причем $\mathcal{T}_{M}$ - топологическая структура на $M$, т.е. множество всех открытых подмножеств данного топологического пространства. Для данного топологического пространства определено множество $\mathcal{F}_{M}$ всех замкнутых его подмножеств. Пусть $\left(M, \mathcal{T}_{M}\right)$ и $\left(Z, \mathcal{T}_{Z}\right)$ топологические пространства, причем второе является топологическим подпространством первого; данный факт записываем как $Z<M$.
} 


$$
\begin{array}{ll}
\chi_{2}(U)=1 ; & \chi_{2}(V)=0 ; \\
\chi_{3}(U)=0 ; & \chi_{3}(V)=1 ; \\
\chi_{4}(U)=0 ; & \chi_{4}(V)=0 ;
\end{array}
$$

Определим отображение $\vartheta: \operatorname{Im} \varphi \rightarrow\{0,1\}^{3}$, такое, что для каждого $R_{k} \in \operatorname{Im} \varphi$

$$
\vartheta\left(R_{k}\right)=\left(\chi_{k}(U), \chi_{k}(V), i\left(t_{k}\right)\right) .
$$

причем $i$ - тождественное отображение на $\{0,1\}$, а $t_{k}=\pi_{2}\left(R_{k}\right)$, где $\pi_{2}: \operatorname{Im} \varphi \rightarrow\{0,1\}$ - проекция $R_{k}$ на $\{0,1\}$.

Определим отображение $\psi: \mathcal{T}_{Y} \cup \mathcal{F}_{Y} \rightarrow\{0,1\}^{3}$, такое, что $\psi=\vartheta \circ \varphi$. Поскольку область определения и множество значений $\vartheta$ имеют одинаковую кардинальность, следует, что $\vartheta$ есть взаимно однозначное отображение; поэтому и $\psi$ является взаимно однозначным отображением множества $\mathcal{T}_{Y} \cup \mathcal{F}_{Y}$ на множество

$$
\operatorname{Im} \psi=\operatorname{Im} \vartheta=\{(1,1,1),(1,0,1),(0,1,0),(0,0,1)\} .
$$

Заметим, что $\operatorname{Im} \psi$ содержит все элементы, принадлежащие графу булевой функции формулы $P \rightarrow Q$, и только их. Притом, первая координата любой точки графа соответствует значению формулы $Q$ для данной интерпретации, вторая координата соответствует значению формулы $P$ для той же интерпретации, а третья - значению интерпретации материальной импликации.

СлеДСтвиЕ 1. Пусть $A, B$ - различные формулы логики высказываний, $A \rightarrow B$ - формула, и $X-$ непустое множество, такое, что $A \in \mathrm{X}$ и $B \in \mathrm{X}$. Тогда булевой функцией $\Pi:\{0,1\}^{2} \rightarrow\{0,1\}$ формулы $A \rightarrow B$ индуцирована топология $\mathcal{T} \rightarrow$ на множестве $\{A, B\} \subseteq X$, такая что $\left(\{A, B\}, \mathcal{T}_{\rightarrow}\right)$ является топологически связным пространством.

ДокАЗАТЕЛЬСТво. Графом функции П является множество

$$
\begin{aligned}
\Gamma_{\Pi} & =\{(I(A), I(B), I(A \rightarrow B)) \mid I \in K\}= \\
& =\{(1,1,1),(0,1,1),(1,0,0),(0,0,1)\},
\end{aligned}
$$

где $K$ - множество всех интерпретаций пропозициональных переменных $A$ и $B$. Каждой интерпретации $I \in K$ присваиваем функцию $\mu_{I}:\{A, B\} \rightarrow \mathcal{P}(X)$ такую, что 


$$
\mu_{I}(N)=\left\{\begin{array}{c}
\{N\}, I(N)=1 \\
\emptyset, I(N)=0
\end{array} ; N \in\{A, B\}\right.
$$

Определим отображение $\lambda: \Gamma_{\Pi} \rightarrow \mathcal{P}(X) \times\{0,1\}$ такое, что

$$
\lambda(I(A), I(B), I(A \rightarrow B))=\left(\mu_{I}(A) \cup \mu_{I}(B), i(I(A \rightarrow B))\right) ; I \in K
$$

причем $i$ является тождественным отображением на множестве значений интерпретаций данной формулы.

Тогда: $\operatorname{Im} \lambda=\{(\{A, B\}, 1),(\{B\}, 1),(\{A\}, 0),(\emptyset, 1)\}$.

Из $|X| \geq 2$ следует существование $G \subset \mathcal{P}(X)$ и $H \subset \mathcal{P}(X)$, таких, что $\emptyset \notin\{G, H\}$ и $G \cap H=\emptyset$. Определим отображение $\alpha: \operatorname{Im} \lambda \rightarrow G \cup H$, такое, что $\alpha(U, p)=U \in G$ для $p=1, \alpha(U, p)=U \in H$ для $p=0$. Получаем: $\{\{A, B\},\{B\}, \emptyset\} \subseteq G$ и $\{\{A\}\} \subseteq H$.

Определим $\mathcal{T}_{\rightarrow}=\{\{A, B\},\{B\}, \emptyset\}$. Таким образом, задана топология $\mathcal{T}_{\rightarrow}$ на множестве $\{A, B\} \subseteq X$ и $\left(\{A, B\}, \mathcal{T}_{\rightarrow}\right)$ является топологическим пространством. Очевидно, нельзя разбить $\left(\{A, B\}, \mathcal{T}_{\rightarrow}\right)$ на два непустых непересекающихся открытых подмножества, т.е. $\left(\{A, B\}, \mathcal{T}_{\rightarrow}\right)$ есть связное топологическое пространство.

Вышеприведенные рассуждения позволили выявить связность топологический инвариант - как необходимый признак моделей формул, главной связкой которых является материальная импликация. В Теореме 1, в частности, доказано, что каждое топологически связное пространство, которое может быть разбито на два собственных подмножества, из которых одно является открытым (и не замкнутым), а другое замкнутым (и не открытым), допускает логическую трактовку в виде кондициональной формулы из условий теоремы (в Следствии доказано обратное утверждение).

Заметим также, что если условия Теоремы 1 выполняются тривиально, т.е. если рассматривается пространство с антидискретной топологией, то через отображение $\psi$ получается булеан материальной импликации, в качестве антецедента и консеквента которой выступает одна и та же формула. Наоборот, если последняя рассматривается с точки зрения Следствия к Теореме 1 , то получается пространство с антидискретной топологией, которое тривиальным образом обладает инвариантом топологической связности.

В дальнейшем приведем топологическую характеризацию правила вывода modus ponens. 
ОПРЕДЕЛЕНИЕ 1. Пусть $L$ непустое конечное множество формул логики высказываний. Через $K_{L}$ обозначим множество всех формул $L$, главной связкой которых является кондиционал ${ }^{8}$, и формул логики высказываний, построенных связыванием элементов $L$ кондиционалом.

Tеорема 2. Пусть $L=\left\{A_{n} \mid n \in \mathbb{N}\right\}$ непустое конечное множество формул логики высказываний, $A$ и В элементы $K_{L} u \mathcal{T}_{A}, \mathcal{T}_{B}, \mathcal{T}_{A \rightarrow B}-$ топологии, индуцированные соответствующими формулами $A, B$, $A \rightarrow B$, в смысле $\mathcal{T}_{\rightarrow}$. Тогда существует отображение $\sigma_{A}:\{A, B\} \rightarrow$ $\cup_{A_{i} \in K_{L}}\left\{A_{i},\left\{\left\{A_{i}\right\}\right\}\right\}$, такое, что для пары $F, \sigma_{F}$ определен гомеоморфизм $\mathcal{M}$ пространств $\left(\{A, B\}, \mathcal{T}_{A \rightarrow B}\right)$ u $\left(\operatorname{Im}_{F}, \mathcal{T}_{E}\right) ; F, E \in\{A, B\}$, который индуцирует формулу $B$, если и только если $F=A$ и $E=B .^{9}$

ДоказательСтво. Обозначим $X=\{A, B\}$. Формула $A$ индуцирует связное топологическое пространство $Y_{A}=\{A,\{\{A\}\}\}$ с топологией $\mathcal{T}_{A}=\{\{A,\{\{A\}\}\},\{A\}, \emptyset\}$. Определим отображение

$$
\begin{gathered}
\sigma_{A}: X \rightarrow \cup_{A_{i} \in K_{L}}\left\{A_{i},\left\{\left\{A_{i}\right\}\right\}\right\} \text { такое, что } \\
\sigma_{A}(A)=\left\{\{A\}_{X}^{c}\right\}=\{\{B\}\} \text { и } \sigma_{A}(B)=B .
\end{gathered}
$$

В таком случае $\operatorname{Im} \sigma_{A}=\{B,\{\{B\}\}\}$. Топология $\mathcal{T}_{A}$ очевидным образом индуцирует топологию $\mathcal{T}_{B}=\{\{B,\{\{B\}\}\},\{B\}, \emptyset\}$ на $\operatorname{Im} \sigma_{A}$.

Определим отображение $\mathcal{M}: X \rightarrow \operatorname{Im} \sigma_{A}$ так, что $\mathcal{M}(A)=\sigma_{A}(A)$ и $\mathcal{M}(B)=\sigma_{A}(B)$. Очевидно, что отображение $\mathcal{M}$ является гомеоморфизмом и связное топологическое пространство $\left(\operatorname{Im}_{A}, \mathcal{T}_{B}\right)$ индуцирует подформулу $B$ формулы $A \rightarrow B$ (Теорема 1 ).

Теперь пусть $\sigma_{B}: X \rightarrow \cup_{A_{i} \in K_{L}}\left\{A_{i},\left\{\left\{A_{i}\right\}\right\}\right\}$ есть отображение, такое, что $\sigma_{B}(A)=A$ и $\sigma_{B}(B)=\left\{\{B\}_{X}^{c}\right\}=\{\{A\}\}$. Областью значения $\sigma_{B}$ является $\operatorname{Im} \sigma_{B}=\{A,\{\{A\}\}\}$ и $\mathcal{T}_{B}$ индуцирует топологию $\mathcal{T}_{A}=\{\{A,\{\{A\}\}\},\{A\}, \emptyset\}$ на $\operatorname{Im} \sigma_{B}$

Далее, определим отображение $\mathcal{N}: X \rightarrow \operatorname{Im} \sigma_{B}$, такое, что

$$
\mathcal{N}(A)=\sigma_{B}(A) \text { и } \mathcal{N}(B)=\sigma_{B}(B) .
$$

Отсюда для

$$
\{A\} \in \mathcal{T}_{A}, \mathcal{N}^{-1}(\{A\})=\{A\} \notin \mathcal{T}_{A \rightarrow B} ;
$$

поскольку $\mathcal{N}$ не является непрерывным отображением пространств, $\mathcal{N}$ не есть гомеоморфизм.

\footnotetext{
${ }^{8}$ Материальная импликация.

${ }^{9} \mathrm{~B}$ этой теореме используем эквивалентность формул $A$ и $(A \rightarrow A) \rightarrow A$ логики высказываний.
} 
Докажем теперь, что гомеоморфизм $\mathcal{M}$ пространств, удовлетворяющих условиям Теоремы 2 , сохраняет истинность ${ }^{10}$.

Пусть $I \in K$ есть интерпретация, такая, что

$$
I(A)=1 \text { и } I(A \rightarrow B)=1 .
$$

Из Следствия к Теореме 1 следует, что функцией $\lambda_{1}$ на графе $\Gamma_{1}$ булевой функции формулы $(A \rightarrow A) \rightarrow A$ и элементами индуцированного ею топологического пространства определено множество

$$
F=\{(\{A\}, 1),(\{A,\{\{A\}\}\}, 1)\} \subset \operatorname{Im} \lambda_{1} .
$$

Соответственно функцией $\lambda_{2}$ на графе $\Gamma_{2}$ булевой функции формулы $A \rightarrow B$ и элементами индуцированного ею топологического пространства определено множество

$$
G=\{(\{B\}, 1),(\{A\} \cup\{B\}, 1),(\emptyset, 1)\} \subset \operatorname{Im} \lambda_{2} .
$$

Из определения функции $\chi$ (Теорема 1 ) следует, что $F$ и $G$ однозначно определяют значения $\chi(A)=1$ и $\chi(B)=1$ соответственно и множество $H=\{(\{B\}, 1),(\{B,\{\{B\}\}\}, 1)\}$ для топологического пространства $\left(\operatorname{Im} \sigma_{A}, \mathcal{T}_{B}\right)$, которое индуцирует подформулу $B$ формулы $A \rightarrow B$. Множеством $H$ однозначно определено значение $I(B)=1$ подформулы $B$ (Теорема 1, Следствие). Утверждение доказано.

Таким образом, упорядоченная тройка $\left(X, \sigma_{A}, \mathcal{M}\right)$ топологического пространства $X=\left(\{A, B\}, \mathcal{T}_{A \rightarrow B}\right)$, функции $\sigma_{A}$ и гомеоморфизма $\mathcal{M}$ представляют топологическое описание правила вывода modus ponens.

\section{4. Топологическая характеризация элементов логической структуры}

Импликативная связка в Теореме 1 индуцирована топологически, путем дифференциации топологически связного пространства. Во-первых, это означает, что в данном случае импликация определяется конструктивно (через топологические свойства дифференцируемого объекта) и, вовторых, такое топологическое описание снабжает импликацию релевантным характером и устраняет известные парадоксы, свойственные

\footnotetext{
${ }^{10}$ Конечно, имеется в виду, что $\mathcal{M}$ ставит в отношение топологические представления логических объектов таким образом, что репрезентируемое $\mathcal{M}$ (мета)логическое отношение между соответствующими логическими представляемыми обладает свойством сохранения истинности.
} 
материальной импликации. Последнее свойство следует из конструктивности поскольку, для получения кондиционала в Теореме 1 нуждаемся в двухкомпонентном топологически связном пространстве (т.е. связь между антецедентом и консеквентом опирается на топологические свойства соответствующего объекта).

С другой стороны, Теорема 1 вместе со следствием к ней утверждают эквивалентность двухкомпонентного топологически связного пространства (из условий Теоремы 1) и материальной импликации, т.е., с формальной точки зрения, каждый кондиционал с различными антецедентом и консеквентом индуцирует одно двухкомпонентное топологически связное пространство. Отсюда следует, что любые высказывания, соединенные кондиционалом, образуют топологически связное пространство данного типа (в соответствии с полученным описанием), а значит упомянутые парадоксы материальной импликации не устраняются.

Для разрешения возникшей проблемы необходимо вернуться к понятию схемы дифференциации и разъяснить решающее значение, которое данного рода схемы играют в этом вопросе.

$$
* * *
$$

Но прежде чем продолжить начатое рассмотрение, сделаем небольшое отступление с целью обобщения тех методологических принципов, которые были использованы в предыдущих примерах для получения топологического описания элементов логических структур.

В третьем параграфе мы ввели термин - инвариант дифференциации, относящийся к формальным элементам, поставленным в соответствие тем аспектам дифференцируемой сущности, которые выделяются в результате применения определенных критериев дифференциации.

В общем случае, уровень структурированности инвариантов дифференциации зависит от разных факторов, имеющих отношение к целям и возможностям моделирования дифференцируемой сущности. Так, например, символы переменных в «Языке переменных» В.И. Шалака можно рассматривать в качестве инвариантов дифференциации данного языка как дифференцируемой сущности, в соответствии с критерием дифференциации, выраженным через требование различения элементов алфавита.

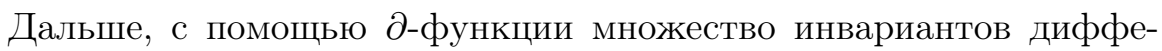
ренциации данной сущности-объекта снабжается топологической структурой. Самый простой и одновременно общий случай заключается в 
снабжении множества (неструктурированных) инвариантов дифференциации дискретной топологией (пространство $V a r$ на множестве знаков языка переменных В.И. Шалака). Введением дополнительных критериев в исходную схему дифференциации данного объекта, позволяющих, например, дифференциацию отношений инвариантов (дифференциации), пространственная структура, характеризующая сущность, меняется, т.е. инварианты дифференциации образуют пространство, которому в общем случае свойственны иные топологические инварианты (в качестве примера последнего может послужить топологическое преобразование дискретного топологического подпространства на Var в пространство с антидискретной топологией в результате введения протологического следования на множестве $V a r$ в том же «Языке переменных»).

Вернемся теперь к начальной проблеме этого параграфа. В случае, когда в итоге дифференциации некоторой сущности, осуществленной применением такой схемы дифференциации, критерии которой относятся к (между прочим) ее нелогическим аспектам (например, к элементам нелогического содержания некоторого языкового контекста), на множестве инвариантов дифференциации индуцируется топологическое пространство, удовлетворяющее условиям Теоремы 1, тогда, в соответствии с теоремой, полученные или производные из результатов дифференциации смысловые комплексы могут быть представлены в виде (пропозициональных) переменных и поставлены в отношение с использованием импликативной связки.

Отсюда понятно, что в данном случае исходные топологические пространства образуются конструктивно, в результате проведения дифференциации конкретных сущностей с использованием определенной схемы дифференциации. Очевидно, что в рассматриваемом случае полученное импликативное отношение между упомянутыми (пропозициональными) переменными или формулами обладает релевантным характером ${ }^{11}$. Релевантность обеспечена на топологических основаниях тем, что одно из топологических пространств, поставленных в соответствие этим формам, содержит только те инварианты дифференциации,

\footnotetext{
${ }^{11}$ Обращаем внимание на то, что говоря о релевантном характере упомянутого импликативного отношения, имеем в виду наличие, в данном случе, содержательной связи между антецедентом и консеквентом. Последнее требование принимаем в качестве необходимого критерия релевантности.
} 
которые содержатся и в другом, т.е. является его подпространством. Таким образом, в данном случае обеспечена содержательная связь между антецедентом и консеквентом. В итоге, данное топологическое представление позволяет обойти известные парадоксы материальной импликации.

С другой стороны, в Следствии к Теореме 1 представлен результат дифференциации материальной импликации в соответствии с только что изложенными началами топологического представления. Критериями дифференциации в этом случае выступают семантические свойства, определяющие связку кондиционала в рамках логики высказываний (аналогичный результат можно получить исходя не из двузначной семантики логики высказываний, а отправляясь от общего понятия модели для классической импликативной связки).

Существенным здесь является тот факт, что топологическая связность как топологический инвариант полученной пространственной структуры, является не характеристикой смысловых комплексов, формально поставленных в отношение связкой кондиционала (т.е. нелогического содержания возможных интерпретаций формул А и В, которое остается вне рамок применяемой в данном случае схемы дифференциации), а характеристикой логической формы, представляющей объект дифференциации (т.е. именно материальной импликации).

Иными словами, в Следствии к Теореме 1 объектом дифференциации является сама материальная импликация (как отношение между элементами соответствующей логической структуры), в качестве критериев дифференциации которой принимаются определяющие ее семантические свойства. Возможное нелогическое содержание формул $A$ и $B$, определяющее соответствующий характер их отношения, остается в данном случае за рамками примененной схемы дифференциации.

В Теореме 2 логическая форма классической импликативной связки дифференцируется дополнительно, по отношению к синтаксическим ее свойствам (т.е. путем введения в схему ее дифференциации критериев, относящихся к последним).

Учитывая вышесказанное, становится понятным, что парадоксы материальной импликации не устраняются на уровне ее топологического описания, данного в Следствии к Теореме 1, т.е. в случае, когда само отношение материальной импликации становится объектом дифференциации согласно критериям, охватывающим ее семантические (и синтаксические) определяющие. Это вполне закономерно, поскольку данные парадоксы и обусловлены последними и в любой адекватной фор- 
мальной характеризации должны возникать тем или иным образом. В нашем случае они высказываются в возможности именно формального образования топологически связных пространств, т.е. возможности их образования без учета внутренней структуры их компонентов.

\section{5. Заключение}

В рамках предварительного и пока далекого от законченности представления подхода к применению топологического аппарата для репрезентации логических объектов необходимо упомянуть и проблему, связанную с топологическим представлением отрицания.

Исходя из вышесказанного, нам представляется, что операцию отрицания можно соотнести с отображением на топологических пространствах (получаемых следуя предыдущим указаниям), которое каждому пространству меняет топологическую структуру, причем, таким образом, что из соответствующего пространства устраняются некоторые инварианты дифференциации (элементы пространства) - через простое удаление или путем соответствующих структурных изменений - или некоторые топологические инварианты структуры самого пространства.

Таким образом, предоставляется возможность формального определения различных вариантов отрицания, свойственных различным логическим системам. Характеризация полученных вариантов отрицания проводится в терминах свойств соответствующих пространств, что позволяет одновременно устанавливать и взаимосвязи между данными вариантами, и переходы от одного определения к другому в качестве отображений между их топологическими представлениями.

В той мере, в которой свойства самой логической системы определяются тем или иным вариантом отрицания, пространственные (топологические) характеристики этих вариантов (а также свойства их отображений) будут способствовать формальному осуществлению взаимосвязей между соответствующими логическими системами на уровне их топологических представлений.

Из всего сказанного очевидно, что топологические репрезентации логических объектов, индуцированные применением различных схем дифференциации, могут, для одних и тех же объектов, быть различными, и эти различные варианты зависят от содержания и структуры самих схем дифференциации рассматриваемых объектов, т.е. от рамок 
контекстуального подхода к ним. Данная особенность описанного приема представляется нам особо важным его свойством.

Топологический формализм предоставляет достаточно наглядное и эффективное средство для осуществления переходов между различными описаниями одного и того же логического объекта, а также между описаниями различных объектов, с целью выявления их взаимосвязей.

Общий «пространственный характер» всего дифференцируемого (параграфы 2 и 3) и применимость выразительных средств топологии к множествам инвариантов дифференциации казусов последнего позволяют приступить к созданию систем топологического описания, в которых, в рамках единой формальной установки, в принципе возможна артикуляция объектного уровня логических систем и их метауровневое рассмотрение и описание в терминах топологических свойств.

\section{Литература}

[1] Бескова И.А. Рифы логико-методологического анализа истинности // Многомерность истины / Под ред. А.А. Горелова, М.М. Новосёлова. М.: ИФ РАН, 2008. С. $162-181$.

[2] Генисаретский О.И. Пространственность в иконологии и эстетике священника Павла Флоренского // Свящ. Павел Флоренский. Статьи и исследования по истории и философии искусства и археологии / Под ред. иг. Андроника (А.С. Трубачева). М.: Мысль, 2000. С. 9-46.

[3] Флоренский П.А. Анализ пространственности (и времени) в художественно-изобразительных произведениях // Свящ. Павел Флоренский. Статьи и исследования по истории и философии искусства и археологии / Под ред. иг. Андроника (А.С. Трубачева). М.: Мысль, 2000. C. $81-259$.

[4] Флоренский П.А. Значение пространственности // Свящ. Павел Флоренский. Статьи и исследования по истории и философии искусства и археологии / Под ред. иг. Андроника (А.С. Трубачева). М.: Мысль, 2000. C. $272-274$.

[5] Шалак В.И. Протологика: новый взгляд на природу логического: Автореф. дис. . д д-ра филос. наук. М.: ИФ РАН, 2010. 42 с.

[6] Johansen J.D., Larsen S.E. Uvod u semiotiku. Zagreb: Croatialiber, 2000. $360 \mathrm{pp}$.

[7] Munkres J.R. Topology. Upper Saddle River: Prentice Hall, 2000. 537 pp.

[8] Rodin A. Axiomatic Method and Category Theory. Springer, 2014. 285 pp.

[9] Rosen J. Symmetry in science. N.Y.: Springer-Verlag, 1995. 213 pp. 


\title{
A.B. BANOVAC
}

\section{Topological Representation of Material Implication and the Rule of Inference Modus Ponens}

\author{
Banovac Andrej Borisovich \\ Institute of Philosophy, Russian Academy of Sciences. \\ 14/5 Volkhonka St., Moscow, 119991, Russian Federation. \\ E-mail: pochtamoya@bk.ru \\ In the present paper we introduce and elaborate some basic elements of the \\ methodological approach characterized by the application of topological means in \\ the analysis and representation of entities through the actualization of some explicit \\ differentiation scheme (scheme of discernment). The latter is understood as a set \\ of rules - termed differentiation criteria - that individuate particular invariants \\ (symmetries) of the entity under examination. We introduce the notion of differentiation \\ invariants as symbolic representatives of the latter, and show that, in all instances \\ of differentiation, topological structure can be induced on the set of such invariants. \\ Given that, we proceed in describing the theoretical framework within which objects \\ of logical theories and systems, as well as properties and interrelations between them, \\ can be represented and treated by formal means of topology and characterized in \\ terms of topological properties. Exposition of the proposed method is given through \\ its application, resulting in topological representation of material implication and the \\ rule of inference modus ponens.
}

Keywords: differentiation, differentiation invariants, Kronecker delta function, topological space, topological representation, topological invariants, material implication, modus ponens

\section{References}

[1] Beskova, I.A. "Rify logiko-metodologicheskogo analiza istinnosti" [Reefs of logical and methodological analysis of the truth], Mnogomernost' istiny [Multidimensionality of truth], ed. by A.A. Gorelova, M.M. Novoselov. Moscow: IF RAS, 2008, pp. 162-181. (In Russian)

[2] Genisaretskii, O.I. "Prostranstvennost' v ikonologii i estetike svyashchennika Pavla Florenskogo" [Spatiality in iconology and aesthetics of priest Paul Florensky], Svyashch. Pavel Florenskii. Stat' $i$ i issledovaniya po istorii $i$ filosofii iskusstva $i$ arkheologii [Priest. Paul Florensky. Articles and studies on the history and philosophy of art and archeology], ed. by ig. Andronicus (A.S. Trubachov). Moscow: Mysl' Publ., 2000, pp. 9-46. (In Russian)

[3] Florenskii, P.A. "Analiz prostranstvennosti (i vremeni) v khudozhestvennoizobrazitel'nykh proizvedeniyakh" [Analysis of the spatiality (and time) in art 
and fine work], Svyashch. Pavel Florenskii. Stat'i i issledovaniya po istorii $i$ filosofii iskusstva $i$ arkheologii [Priest. Paul Florensky. Articles and studies on the history and philosophy of art and archeology], ed. by ig. Andronicus (A.S. Trubachov). Moscow: Mysl' Publ., 2000, pp. 81-259. (In Russian)

[4] Florenskii, P.A. "Znachenie prostranstvennosti" [The value of spatiality], Svyashch. Pavel Florenskii. Stat'i i issledovaniya po istorii i filosofii iskusstva $i$ arkheologii [Priest. Paul Florensky. Articles and studies on the history and philosophy of art and archeology], ed. by ig. Andronicus (A.S. Trubachov). Moscow: Mysl' Publ., 2000, pp. 272-274.(In Russian)

[5] Shalack. V.I., Protologika: novyi vzglyad na prirodu logicheskogo [Protologika: a new look at the nature of logic]. Avtoref. dis. ... d-ra filos. nauk. Moscow: IF RAS, 2010. 42 pp. (In Russian)

[6] Johansen, J.D., Larsen, S.E. Uvod u semiotiku. Zagreb: Croatialiber, 2000. 360 pp.

[7] Munkres, J.R. Topology. Upper Saddle River: Prentice Hall, 2000. 537 pp.

[8] Rodin, A. Axiomatic Method and Category Theory. Springer, 2014. 285 pp.

[9] Rosen, J. Symmetry in science. N.Y.: Springer-Verlag, 1995. 213 pp. 